\title{
THE TRANSCENDENCE DEGREE OF AN INTEGRAL DOMAIN OVER A SUBFIELD AND THE DIMENSION OF THE DOMAIN
}

\author{
HIROSHI TANIMOTO
}

To the memory of Professor Hideyuki Matsumura

\begin{abstract}
For a domain $A$ containing a field $k$ with $\operatorname{tr} \cdot \operatorname{deg}_{k} A<\infty$, we define a new transcendence degree of $A$ with respect to $k$, which is denoted by $\operatorname{td}_{k} A$. By using this, we generalize the theorem that for every affine domain $A$ over a field $k$ it holds that $\operatorname{dim} A=\operatorname{tr} \cdot \operatorname{deg}_{k} A$. For example, we show that if $A$ is a quasi-local domain containing a field $k$ with $\operatorname{dim} A=\operatorname{td}_{k} A<\infty$, then for every Noetherian local $k$-subalgebra $R$ of $A$ it holds that $\operatorname{dim} R=\operatorname{td}_{k} R$. Moreover we also generalize the theorem due to Gilmer, Nashier and Nichols.
\end{abstract}

\section{$\S 1$. Introduction}

The following theorem is well-known.

TheOREm A. Let $k[\underline{a}]=k\left[a_{1}, \ldots, a_{n}\right]$ be an affine domain over a field $k$. Then it holds that $\operatorname{dim} k[\underline{a}]=\operatorname{tr} \cdot \operatorname{deg}_{k} k[\underline{a}]$.

A good many people have tried to generalize this theorem. We take up two of their theorems.

In 1982, Onoda and Yoshida obtained the following theorem.

Theorem B. ([7]) Let $A$ be an affine domain over a field $k$. Then for any $k$-subalgebra $R$ of $A$, we have $\operatorname{dim} R=\operatorname{tr} \cdot \operatorname{deg}_{k} R$.

In 1989, Gilmer, Nashier and Nichols obtained the following theorem. They extend the affine domain $A$ in Theorem B to a local domain $A_{\mathfrak{m}}$.

Received May 10, 2001.

Revised January 18, 2002.

2000 Mathematics Subject Classification: 13C15;13E05;13B30. 
Theorem C. ([3]) Let $A$ be an affine domain over a field $k$ and $\mathfrak{m} a$ maximal ideal of $A$. Then for any $k$-subalgebra $R$ of $A_{\mathfrak{m}}$, we have $\operatorname{dim} R=$ tr. $\operatorname{deg}_{k} R$.

In fact, this theorem is proved by using the following Theorem $\mathrm{A}^{\prime}$, a weaker form of Theorem A.

TheOrem $\mathrm{A}^{\prime}$. Let $A$ be an affine domain containing a field $k$. Then for any maximal ideal $\mathfrak{m}$ of $A$, it holds that $\operatorname{dim} A_{\mathfrak{m}}=\operatorname{tr} \cdot \operatorname{deg}_{k} A_{\mathfrak{m}}$.

We want to make the same attempt as they did. For example, in Theorem $\mathrm{A}^{\prime}$ we want to change the ideal to any prime ideal $\mathfrak{p}$. But if we were to consider the transcendence degree of $A_{\mathfrak{p}}$ over $k$, we would fail. Hence first of all we have to extend the definition of the transcendence degree over a field. So in Section 2, for a domain $A$ containing a field $k$ with $\operatorname{tr} \cdot \operatorname{deg}_{k} A<\infty$ we define a new transcendence $\operatorname{degree} \operatorname{td}_{k} A$ by $\min \left\{\operatorname{tr} \cdot \operatorname{deg}_{\ell} A \mid \ell\right.$ is a subfield of $A$ with $\left.\ell \supseteq k\right\}$. The new transcendence degree is a natural generalization of the usual one, as stated in Section 2. In this paper, by using $\operatorname{td}_{k} A$, we shall generalize Theorem $\mathrm{A}^{\prime}$ and Theorem C. In particular, to generalize Theorem $\mathrm{C}$, we set the following problem:

Let $A$ be a quasi-local domain containing a field $k$ with $\operatorname{tr} \cdot \operatorname{deg}_{k} A<\infty$ ( $\star$ such that $\operatorname{dim} A=\operatorname{td}_{k} A$. Then, for every $k$-subalgebra $R$ of $A$, does it hold that $\operatorname{dim} R=\operatorname{td}_{k} R$ ?

We consider the problem $(\star)$ above in the following two cases:

Case A $R$ is Noetherian;

Case B $A$ is Noetherian.

In Section 3, we study a Noetherian catenary domain, and get the following result, which is a generalization of Theorem $\mathrm{A}^{\prime}$.

Theorem 3.1. Let $A$ be a Noetherian domain and $k$ a subfield of $A$ with tr. $\operatorname{deg}_{k} A<\infty$. Assume that $\operatorname{dim} A_{\mathfrak{m}}=\operatorname{td}_{k} A_{\mathfrak{m}}$ for any $\mathfrak{m} \in \operatorname{Max} A$. Then the following conditions are equivalent:

(1) A is catenary;

(2) for any $\mathfrak{m} \in \operatorname{Max} A$ and any $\mathfrak{p} \in \operatorname{Spec} A$ with $\mathfrak{m} \supseteq \mathfrak{p}$,

(a) $\operatorname{dim} A_{\mathfrak{p}}=\operatorname{td}_{k} A_{\mathfrak{p}}$, and

(b) $\operatorname{dim} A_{\mathfrak{m}} / \mathfrak{p} A_{\mathfrak{m}}=\operatorname{td}_{k} A_{\mathfrak{m}} / \mathfrak{p} A_{\mathfrak{m}}$;

(3) for any $\mathfrak{p}, \mathfrak{q} \in \operatorname{Spec} A$ with $\mathfrak{p} \supseteq \mathfrak{q}, \operatorname{dim} A_{\mathfrak{p}} / \mathfrak{q} A_{\mathfrak{p}}=\operatorname{td}_{k} A_{\mathfrak{p}} / \mathfrak{q} A_{\mathfrak{p}}$. 
In Section 4, we study the problem $(\star)$. In Case A, the following theorem is proved.

TheOREM 4.1. Let $(R, \mathfrak{m})$ be a Noetherian local domain, $(A, \mathfrak{n})$ a quasilocal domain dominating $R$ and $k$ a subfield of $R$ with $\operatorname{tr} \cdot \operatorname{deg}_{k} A<\infty$. Then $\operatorname{td}_{k} R-\operatorname{dim} R \leq \operatorname{td}_{k} A-\operatorname{dim} A$.

From this theorem, we get Corollary 4.1 at once, which is an affirmative answer to the problem $(\star)$ in the case that $R$ is local. But if $R$ is not local, the answer is negative. For the fact, see Example 6.6. In Case B, from Example 4.1 we see that the answer is negative in general. But if the field $k$ is a "tm-subfield" of $A$, which is defined in Section 2, and if $A$ is catenary, the answer is affirmative. Concerning the fact, see Theorem 4.3, which is a natural generalization of Theorem C. In Section 5, for a semigroup ring $k[M]$ over a field $k$, where $M$ is a semigroup generated by monomials in finite variables, we show that our equality holds by constructing a chain of prime ideals of length tr. $\operatorname{deg}_{k} k[M]$. For this fact, see Theorem 5.1. Note that $M$ may be generated by infinite elements and that the powers of variables in each monomial may be rational. In Section 6, we give some examples. For instance, Example 6.3 shows that $\operatorname{td}_{k} A$ does not have a local property in general, that is, there is a regular domain $A$ containing a field $k$ such that $\operatorname{td}_{k} A>\max \left\{\operatorname{td}_{k} A_{\mathfrak{m}} \mid \mathfrak{m}\right.$ is a maximal ideal of $\left.A.\right\}$.

In this paper, all rings are assumed to be commutative and unitary, and for a domain $A$ we denote the set of the maximal ideals of $A$ by $\operatorname{Max} A$ and the total quotient field of $A$ by $Q(A)$. Moreover the set of the integers and the set of the rational numbers are denoted by $\mathbf{Z}$ and $\mathbf{Q}$, respectively. For the other notation and terminology, we follow those of [5] and [6].

The author expresses his hearty thanks to Professor H. Uda for his many useful suggestions and encouragements. And the author also expresses his hearty thanks to the referee, whose advice made the proofs of Example $6.1,6.2$ and 6.3 very concise. In particular, the polynomials $f_{a}(X, Y)$ in Example 6.3, by which the proof became very easy, are due to the referee.

\section{§2. A new transcendence degree of a domain containing a field}

For example, let $A$ be an affine domain over a field $k$ and $\mathfrak{p}$ any prime ideal of $A$. When we consider whether $\operatorname{dim} A_{\mathfrak{p}}$ is equal to the transcendence degree of $A_{\mathfrak{p}}$ over a subfield, over what subfield should we consider the transcendence degree? If $\mathfrak{p}$ is not a maximal ideal, the subfield need to be something but $k$. For the problem, we note the following lemma. 
LEMma 2.1. ([3, Proposition 1.1]) Let $R$ be a domain containing a field $k$. Then $\operatorname{dim} R \leq$ tr. $\operatorname{deg}_{k} R$.

From this, it is enough to consider the maximal subfield of $A_{\mathfrak{p}}$ over which the transcendence degree of $A_{\mathfrak{p}}$ is the minimum in the set $\left\{\operatorname{tr} \cdot \operatorname{deg}_{\ell} A_{\mathfrak{p}} \mid\right.$ $\ell$ is a subfield of $\left.A_{\mathfrak{p}}.\right\}$. We notice that we have only to consider the minimum and that we need not be interested in the subfield which gives the minimum. Hence, in general, for a domain $A$ containing a field, we call $\min \left\{\operatorname{tr} \cdot \operatorname{deg}_{\ell} A \mid \ell\right.$ is a subfield of $A$. $\}$ the transcendence degree of $A$ and denote it by $\operatorname{td} A$. Now we hope that for each maximal subfield $\ell$ of $A$, tr.deg $\operatorname{deg}_{\ell} A$ is equal to the minimum. But, as we see in Example 6.1, even if $A$ is a DVR, $\operatorname{tr} \cdot \operatorname{deg}_{\ell} A$ may be a different value for each maximal subfield $\ell$. This makes the situation difficult. Hence we modify the definition of $\operatorname{td} A$ a little.

Let $k$ be a subfield of a domain $A$ with $\operatorname{tr} \cdot \operatorname{deg}_{k} A<\infty$. Then we call $\min \left\{\operatorname{tr} \cdot \operatorname{deg}_{\ell} A \mid \ell\right.$ is a subfield of $A$ with $\left.\ell \supseteq k.\right\}$ the transcendence degree of $A$ with respect to $k$, and denote it by $\operatorname{td}_{k} A$. The new transcendence degree $\operatorname{td}_{k} A$ has good properties and is easy to deal with. For example, if $A$ is quasi-semilocal, then for any maximal subfield with $K \supseteq k$ we have $\operatorname{td}_{k} A=$ tr. $\operatorname{deg}_{K} A$, as we see in Lemma 2.3. Therefore in this paper we deal with $\operatorname{td}_{k} A$. Note that for any domain $A$ containing a field $k$ with tr. $\operatorname{deg}_{k} A<\infty$, we have $\operatorname{dim} A \leq \operatorname{td} A \leq \operatorname{td}_{k} A$. Moreover, note that the transcendence degrees in the theorems in Section 1 are equal to the new transcendence degree. Now, as we see in Example 6.3, there exists a Noetherian domain $A$ such that $\operatorname{dim} A_{\mathfrak{m}}=\operatorname{td}_{k} A_{\mathfrak{m}}$ for any $\mathfrak{m} \in \operatorname{Max} A$ but $\operatorname{dim} A \neq \operatorname{td}_{k} A$. Therefore we find that $\operatorname{td}_{k} A$ tends to have good properties when it is used locally.

Now for a domain $A$ containing a field $k$, where $A$ is not necessarily quasi-semilocal, and for a maximal subfield $K$ of $A$ with $K \supseteq k$, suppose that $\operatorname{td}_{k} A=\operatorname{tr} \cdot \operatorname{deg}_{K} A$. Then for any subfield $L$ of $K$ such that $K$ is algebraic over $L$, we have $\operatorname{td}_{k} A=\operatorname{tr} \cdot \operatorname{deg}_{L} A$. Such a subfield $L$ is so useful that we name it. When a subfield $L$ of a domain $A$ satisfies the condition that for any subfield $K$ of $A$ with $K \supseteq L, K$ is algebraic over $L$, we call $L$ a transcendentally maximal subfield (a tm-subfield, for short) of $A$. Of course, all maximal subfields are tm-subfields.

For a quasi-local domain $A$, a necessary and sufficient condition for a subfield of $A$ to be a tm-subfield is given as follows, which can be proved easily. 
Lemma 2.2. Let $(A, \mathfrak{m})$ be a quasi-local domain containing a field $K$. Then $K$ is a tm-subfield of $A$ if and only if the residue field $A / \mathfrak{m}$ is algebraic over $K$.

For a quasi-semilocal domain, we have a characterization of a tmsubfield as follows.

Lemma 2.3. Let $A$ be a quasi-semilocal domain containing a field $K$. Then $K$ is a tm-subfield of $A$ if and only if $K$ is a tm-subfield of $A_{\mathfrak{m}}$ for some $\mathfrak{m} \in \operatorname{Max} A$.

Proof. It is enough to show the "only if" part. Put $u=\min \left\{\operatorname{tr} . \operatorname{deg}_{K}\right.$ $A / \mathfrak{m} \mid \mathfrak{m} \in \operatorname{Max} A\}$, and assume that $u>0$. Since $A$ is quasi-semilocal, there exists $x \in A$ such that $x \bmod \mathfrak{m}$ is algebraically independent over $K$ for any $\mathfrak{m} \in \operatorname{Max} A$. Then $x$ is also algebraically independent over $K$. Since $K[x] \cap \mathfrak{m}=(0)$ for any $\mathfrak{m} \in \operatorname{Max} A$, we have $K(x) \subseteq A$. This contradicts the fact that $K$ is a tm-subfield of $A$. Hence there exists $\mathfrak{m} \in \operatorname{Max} A$ such that $\operatorname{tr} \cdot \operatorname{deg}_{K} A / \mathfrak{m}=0$, so that $K$ is a tm-subfield of $A_{\mathfrak{m}}$ by Lemma 2.2.

We may ask whether, for every tm-subfield $K$ with $K \supseteq k, \operatorname{td}_{k} A=$ tr. $\operatorname{deg}_{K} A$ holds, or whether $\operatorname{td}_{k} A$ has a local property. If $A$ is quasisemilocal, the answers for these questions are affirmative as follows.

THEOREM 2.1. Let $A$ be a quasi-semilocal domain containing a field $k$ with tr. $\operatorname{deg}_{k} A<\infty$. Then

(1) for any tm-subfield $K$ of $A$ with $\operatorname{tr} \cdot \operatorname{deg}_{K \cap k} k<\infty$, we have $\operatorname{td}_{k} A=$ tr. $\operatorname{deg}_{K} A$;

(2) $\operatorname{td}_{k} A=\max \left\{\operatorname{td}_{k} A_{\mathfrak{m}} \mid \mathfrak{m} \in \operatorname{Max} A\right\}$.

Proof. (1) Let $L$ be a tm-subfield of $A$ with $L \supseteq k$. Then it is enough to show that tr. $\operatorname{deg}_{K} A=\operatorname{tr} \cdot \operatorname{deg}_{L} A$ for any tm-subfield $K$ with tr.deg $K \cap k<\infty$. By Lemma 2.2 and 2.3, there exists a maximal ideal $\mathfrak{m}$ of $A$ such that $A / \mathfrak{m}$ is algebraic over $K$, so that tr.deg $K \cap K=\operatorname{tr} \cdot \operatorname{deg}_{K \cap k} L$. In the same way, we have tr. $\operatorname{deg}_{K \cap k} L \geq \operatorname{tr} \cdot \operatorname{deg}_{K \cap k} K$. Therefore it follows that tr. $\operatorname{deg}_{K \cap k} K=$ tr. $\operatorname{deg}_{K \cap k} L$, so that tr. $\operatorname{deg}_{K} A=\operatorname{tr} \cdot \operatorname{deg}_{L} A$.

(2) Let $K$ be a tm-subfield of $A$ with $K \supseteq k$. Then by Lemma 2.3, $K$ is a tm-subfield of $A_{\mathfrak{m}}$ for some $\mathfrak{m} \in \operatorname{Max} A$. Hence by $(1), \operatorname{td}_{k} A_{\mathfrak{m}}=$ tr. $\operatorname{deg}_{K} A_{\mathfrak{m}}=\operatorname{td}_{k} A$.

Remark 2.1. Theorem 2.1 (2) does not hold in general. In Example 6.3 we get such a negative example. But the author does not know whether Theorem 2.1 (1) holds in general. 


\section{$\S 3$. Catenary domains}

In this section, for each Noetherian domain $A$ containing a field $k$ such that $\operatorname{dim} A_{\mathfrak{m}}=\operatorname{td}_{k} A_{\mathfrak{m}}$ for any $\mathfrak{m} \in \operatorname{Max} A$, we study the question when the domain is catenary or universally catenary. Our results got here will be used in Section 4. First of all, we consider a condition for the domain above to be catenary.

Theorem 3.1. Let $A$ be a Noetherian domain and $k$ a subfield of $A$ with tr. $\operatorname{deg}_{k} A<\infty$. Assume that $\operatorname{dim} A_{\mathfrak{m}}=\operatorname{td}_{k} A_{\mathfrak{m}}$ for any $\mathfrak{m} \in \operatorname{Max} A$. Then the following conditions are equivalent:

(1) A is catenary;

(2) for any $\mathfrak{m} \in \operatorname{Max} A$ and any $\mathfrak{p} \in \operatorname{Spec} A$ with $\mathfrak{m} \supseteq \mathfrak{p}$,

(a) $\operatorname{dim} A_{\mathfrak{p}}=\operatorname{td}_{k} A_{\mathfrak{p}}$, and

(b) $\operatorname{dim} A_{\mathfrak{m}} / \mathfrak{p} A_{\mathfrak{m}}=\operatorname{td}_{k} A_{\mathfrak{m}} / \mathfrak{p} A_{\mathfrak{m}}$;

(3) for any $\mathfrak{p}, \mathfrak{q} \in \operatorname{Spec} A$ with $\mathfrak{p} \supseteq \mathfrak{q}, \operatorname{dim} A_{\mathfrak{p}} / \mathfrak{q} A_{\mathfrak{p}}=\operatorname{td}_{k} A_{\mathfrak{p}} / \mathfrak{q} A_{\mathfrak{p}}$.

Proof. It is enough to show (1) $\Leftrightarrow(2)$. By [5, Theorem 31.4], $A$ is catenary if and only if $\operatorname{dim} A_{\mathfrak{m}}=\operatorname{dim} A_{\mathfrak{p}}+\operatorname{dim} A_{\mathfrak{m}} / \mathfrak{p} A_{\mathfrak{m}}$ for every $\mathfrak{m} \in \operatorname{Max} A$ and every $\mathfrak{p} \in \operatorname{Spec} A$ with $\mathfrak{p} \subseteq \mathfrak{m}$. Hence the result follows from Lemma 2.1 and 3.1 and from the assumption that $\operatorname{dim} A_{\mathfrak{m}}=\operatorname{td}_{k} A_{\mathfrak{m}}$ holds for any $\mathfrak{m} \in \operatorname{Max} A$.

Lemma 3.1. Let $k$ be a subfield of a quasi-local domain $A$ with tr.deg ${ }_{k}$ $A<\infty$. Then we have $\operatorname{td}_{k} A=\operatorname{td}_{k} A_{\mathfrak{p}}+\operatorname{td}_{k} A / \mathfrak{p}$ for any $\mathfrak{p} \in \operatorname{Spec} A$.

Proof. Let $K$ be a tm-subfield of $A$ with $K \supseteq k$. Then $K$ is also a tm-subfield of $A / \mathfrak{p}$ by Lemma 2.2. Put $t=\operatorname{tr} \cdot \operatorname{deg}_{K} A / \mathfrak{p}$. Then there exist $x_{1}, \ldots, x_{t} \in A$ such that $x_{1} \bmod \mathfrak{p}, \ldots, x_{t} \bmod \mathfrak{p}$ are algebraically independent over $K$. Then so are $x_{1}, \ldots, x_{t}$, and $K\left[x_{1}, \ldots, x_{t}\right] \cap \mathfrak{p}=(0)$. Hence $K\left(x_{1}, \ldots, x_{t}\right) \subseteq A_{\mathfrak{p}}$. Then $K\left(x_{1}, \ldots, x_{t}\right)$ is a tm-subfield of $A_{\mathfrak{p}}$ by Lemma 2.2. Hence by Theorem 2.1, we have $\operatorname{td}_{k} A_{\mathfrak{p}}=\operatorname{tr} \cdot \operatorname{deg}_{K\left(x_{1}, \ldots, x_{t}\right)} A_{\mathfrak{p}}=$ tr.deg $\operatorname{deg}_{K} A_{\mathfrak{p}}-\operatorname{tr} . \operatorname{deg}_{K} A / \mathfrak{p}=\operatorname{td}_{k} A-\operatorname{td}_{k} A / \mathfrak{p}$.

Remark 3.1. For the conditions (a) and (b) of Theorem 3.1, we find that there exists a Noetherian local domain which satisfies (a) but does not satisfy (b), as we see in Example 6.4. But the author does not succeed in finding out the example which satisfies (b) but does not satisfy (a).

For a quasi-local domain $(A, \mathfrak{m})$ and any prime ideal $\mathfrak{p}$ of $A$, we set $\gamma(\mathfrak{p})=\operatorname{dim} A-\operatorname{dim} A_{\mathfrak{p}}-\operatorname{dim} A / \mathfrak{p}, \delta(\mathfrak{p})=\operatorname{td}_{k} A_{\mathfrak{p}}-\operatorname{dim} A_{\mathfrak{p}}$ and $\varepsilon(\mathfrak{p})=$ 
$\operatorname{td}_{k} A / \mathfrak{p}-\operatorname{dim} A / \mathfrak{p}$. Note that these values are non-negative integers. Then, by Lemma 3.1, we have the following result.

Corollary 3.1. Let $(A, \mathfrak{m})$ be a quasi-local domain containing a field $k$ with $\operatorname{tr} \cdot \operatorname{deg}_{k} A<\infty$, and let $\mathfrak{p}$ be any prime ideal of $A$. Set $a=\delta(\mathfrak{m})$. Then we have $\delta(\mathfrak{p})+\varepsilon(\mathfrak{p})=\gamma(\mathfrak{p})+a$.

On the other hand, by Theorem 3.1 we can generalize Theorem $\mathrm{A}^{\prime}$ as follows.

Corollary 3.2. For any affine domain $A$ over a field $k$ and for any $\mathfrak{p} \in \operatorname{Spec} A$, we have $\operatorname{dim} A_{\mathfrak{p}}=\operatorname{td}_{k} A_{\mathfrak{p}}$.

In Corollary 3.2, by changing the domain $A$, we can regard the local ring $A_{\mathfrak{p}}$ as the localization of an affine domain by a maximal ideal as follows.

Proposition 3.1. Let $k[\underline{a}]=k\left[a_{1}, \ldots, a_{n}\right]$ be an affine domain over a field $k, \mathfrak{p}$ any prime ideal of $k[\underline{a}]$ and $K$ any tm-subfield of $k[\underline{a}]_{\mathfrak{p}}$ with $K \supseteq k$. Then there exists $\mathfrak{P} \in \operatorname{Max} K[\underline{a}]$ such that $k[\underline{a}]_{\mathfrak{p}}=K[\underline{a}]_{\mathfrak{P}}$.

Proof. Set $\mathfrak{P}=\mathfrak{p} k[\underline{a}]_{\mathfrak{p}} \cap K[\underline{a}]$. Then since $k[\underline{a}] \subseteq K[\underline{a}] \subseteq k[\underline{a}]_{\mathfrak{p}}$, we have $\mathfrak{P} \in \operatorname{Spec} K[\underline{a}]$ and $k[\underline{a}]_{\mathfrak{p}}=K[\underline{a}]_{\mathfrak{P}}$. By Corollary $3.2, \operatorname{dim} K[\underline{a}]_{\mathfrak{P}}=$ $\operatorname{td}_{K} K[\underline{a}]_{\mathfrak{P}}=\operatorname{tr} \cdot \operatorname{deg}_{K} K[\underline{a}]_{\mathfrak{P}}$, so that $\mathfrak{P}$ is a maximal ideal of $K[\underline{a}]$ by Theorem $\mathrm{A}^{\prime}$.

Next, we consider a necessary and sufficient condition for the domain of Theorem 3.1 to be universally catenary.

THEOREM 3.2. Let $k$ be a subfield of a Noetherian domain $A$ with tr. $\operatorname{deg}_{k} A<\infty$. Assume that $\operatorname{dim} A_{\mathfrak{m}}=\operatorname{td}_{k} A_{\mathfrak{m}}$ for any $\mathfrak{m} \in \operatorname{Max} A$. Then $A$ is universally catenary if and only if $\operatorname{dim} B=\operatorname{td}_{k} B$ for every local domain $B$ essentially of finite type over $A$.

Proof. By Theorem 3.1, it is enough to prove the following proposition.

Proposition 3.2. Let $k$ be a subfield of a Noetherian domain $A$ with tr. $\operatorname{deg}_{k} A<\infty$ and $\underline{X}=\left\{X_{1}, \ldots, X_{n}\right\}$ a set of variables over $A$. Let $\mathfrak{P}$ be a prime ideal of $A[\underline{X}]$, and put $\mathfrak{p}=\mathfrak{P} \cap A$. Then if $\operatorname{dim} A_{\mathfrak{p}}=\operatorname{td}_{k} A_{\mathfrak{p}}$, we have $\operatorname{dim} A[\underline{X}]_{\mathfrak{P}}=\operatorname{td}_{k} A[\underline{X}]_{\mathfrak{P}}$. 
Proof. By induction on $n$, we may assume that $n=1$. Put $X=X_{1}$. Moreover we may assume that $A$ is a local domain whose maximal ideal is $\mathfrak{p}$. Then it is enough to show that $\operatorname{dim} A[X]_{\mathfrak{P}} \geq \operatorname{td}_{k} A[X]_{\mathfrak{P}}$.

If $\mathfrak{P}=\mathfrak{p} A[X]$, we have $K[X] \cap \mathfrak{P}=(0)$ for any subfield $K$ of $A$, so that $K(X) \subseteq A[X]_{\mathfrak{P}}$ and tr.deg $K(X) A[X]_{\mathfrak{P}}=\operatorname{tr} \cdot \operatorname{deg}_{K} A$. Hence $\operatorname{dim} A[X]_{\mathfrak{P}}=$ $\operatorname{dim} A=\operatorname{td}_{k} A \geq \operatorname{td}_{k} A[X]_{\mathfrak{P}}$.

Next we assume that $\mathfrak{P} \supset \mathfrak{p} A[X]$. Then for any tm-subfield $K$ of $A$ with $K \supseteq k$, we have $\operatorname{dim} A[X]_{\mathfrak{P}}=\operatorname{dim} A+1=\operatorname{td}_{k} A+1=\operatorname{tr} \cdot \operatorname{deg}_{K} A+1=$ tr.deg $\operatorname{deg}_{K} A[X]_{\mathfrak{P}} \geq \operatorname{td}_{k} A[X]_{\mathfrak{P}}$.

Remark 3.2. Even if we assume that $\operatorname{dim} A_{\mathfrak{m}}=\operatorname{td}_{k} A_{\mathfrak{m}}$ for every $\mathfrak{m} \in$ $\operatorname{Max} A$, being catenary is different from being universally catenary. For such an example, see Example 6.4.

\section{$\S 4$. The problem $(\star)$}

Case A In this case, if $R$ is local, the answer is affirmative as follows.

THEOREM 4.1. Let $(R, \mathfrak{m})$ be a Noetherian local domain, $(A, \mathfrak{n})$ a quasilocal domain dominating $R$ and $k$ a subfield of $R$ with $\operatorname{tr} \cdot \operatorname{deg}_{k} A<\infty$. Then $\operatorname{td}_{k} R-\operatorname{dim} R \leq \operatorname{td}_{k} A-\operatorname{dim} A$.

Proof. Let $K$ be a tm-subfield of $R$ with $K \supseteq k$ and $L$ a tm-subfield of $A$ with $L \supseteq k$, and set $\kappa(\mathfrak{m})=R / \mathfrak{m}$ and $\kappa(\mathfrak{n})=A / \mathfrak{n}$. Then by Lemma 2.2, tr. $\operatorname{deg}_{k} \kappa(\mathfrak{m})=$ tr. $\operatorname{deg}_{k} K$ and tr. $\operatorname{deg}_{k} \kappa(\mathfrak{n})=\operatorname{tr} \cdot \operatorname{deg}_{k} L$. Hence

$$
\begin{array}{rl}
\operatorname{tr} \cdot \operatorname{deg}_{R} & A-\operatorname{tr} \cdot \operatorname{deg}_{\kappa(\mathfrak{m})} \kappa(\mathfrak{n}) \\
& =\left(\operatorname{tr} \cdot \operatorname{deg}_{k} A-\operatorname{tr} \cdot \operatorname{deg}_{k} R\right)-\left(\operatorname{tr} \cdot \operatorname{deg}_{k} \kappa(\mathfrak{n})-\operatorname{tr} \cdot \operatorname{deg}_{k} \kappa(\mathfrak{m})\right) \\
& =\left(\operatorname{tr} \cdot \operatorname{deg}_{k} A-\operatorname{tr} \cdot \operatorname{deg}_{k} L\right)-\left(\operatorname{tr} \cdot \operatorname{deg}_{k} R-\operatorname{tr} \cdot \operatorname{deg}_{k} K\right) \\
& =\operatorname{tr} \cdot \operatorname{deg}_{L} A-\operatorname{tr} \cdot \operatorname{deg}_{K} R \\
& =\operatorname{td}_{k} A-\operatorname{td}_{k} R .
\end{array}
$$

Therefore our claim follows from [2, Theorem A.19].

Corollary 4.1. Let $R$ be a Noetherian local domain, $A$ a quasi-local domain dominating $R$ and $k$ a subfield of $R$ with $\operatorname{tr} \cdot \operatorname{deg}_{k} A<\infty$. Then if $\operatorname{dim} A=\operatorname{td}_{k} A$, we have $\operatorname{dim} R=\operatorname{td}_{k} R$.

In particular, let $T$ be a localization of an affine domain over a field $k$ by a prime ideal, $\bar{T}$ the integral closure of $T$ in the algebraic closure of $Q(T)$ and $\mathfrak{m}$ a maximal ideal of $\bar{T}$ with ht $\mathfrak{m}=\operatorname{dim} T$. Set $A=\bar{T}_{\mathfrak{m}}$. Then for any Noetherian local domain $R$ which contains $k$ and is dominated by $A$, we have $\operatorname{dim} R=\operatorname{td}_{k} R$. 
Proof. For the latter part, we have $\operatorname{dim} T=\operatorname{td}_{k} T$ by Corollary 3.2. Therefore $\operatorname{dim} A=\operatorname{td}_{k} A$. Hence our assertion follows from Theorem 4.1.

Remark 4.1. If $R$ is not local, Corollary 4.1 does not necessarily hold. We give such an example in Example 6.6.

Next we state an application of Corollary 4.1.

TheOREm 4.2. Let $k$ be a field, and let $A$ and $R$ be Noetherian domains such that $k \subseteq R \subseteq A$ and $\operatorname{tr} \cdot \operatorname{deg}_{k} A<\infty$. We assume that $\operatorname{dim} A_{\mathfrak{M}}=$ $\operatorname{td}_{k} A_{\mathfrak{M}}$ for any $\mathfrak{M} \in \operatorname{Max} A$, and that for any $\mathfrak{p} \in \operatorname{Spec} R$ and any $\mathfrak{m} \in$ $\operatorname{Max} R$ with $\mathfrak{p} \subseteq \mathfrak{m}$, there exist $\mathfrak{P}, \mathfrak{Q} \in \operatorname{Spec} A$ such that $\mathfrak{P} \subseteq \mathfrak{Q}, \mathfrak{P} \cap R=\mathfrak{p}$ and $\mathfrak{Q} \cap R=\mathfrak{m}$. Then if $A$ is catenary, so is $R$, and we have $\operatorname{dim} R_{\mathfrak{m}}=$ $\operatorname{td}_{k} R_{\mathfrak{m}}$ for any $\mathfrak{m} \in \operatorname{Max} R$.

Proof. It is enough to show that $R$ satisfies Theorem 3.1 (2). By assumption, for any $\mathfrak{p} \in \operatorname{Spec} R$ and any $\mathfrak{m} \in \operatorname{Max} R$ with $\mathfrak{p} \subseteq \mathfrak{m}$, there exist $\mathfrak{P}, \mathfrak{Q} \in \operatorname{Spec} A$ such that $\mathfrak{P} \subseteq \mathfrak{Q}, \mathfrak{P} \cap R=\mathfrak{p}$ and $\mathfrak{Q} \cap R=\mathfrak{m}$. Then $k \subseteq R_{\mathfrak{p}} \subseteq A_{\mathfrak{P}}$ and $k \subseteq R_{\mathfrak{m}} / \mathfrak{p} R_{\mathfrak{m}} \subseteq A_{\mathfrak{Q}} / \mathfrak{P} A_{\mathfrak{Q}}$. Since $A$ is catenary, it follows from Theorem 3.1 that $\operatorname{dim} A_{\mathfrak{P}}=\operatorname{td}_{k} A_{\mathfrak{P}}$ and $\operatorname{dim} A_{\mathfrak{Q}} / \mathfrak{P} A_{\mathfrak{Q}}=$ $\operatorname{td}_{k} A_{\mathfrak{Q}} / \mathfrak{P} A_{\mathfrak{Q}}$. Hence we have $\operatorname{dim} R_{\mathfrak{p}}=\operatorname{td}_{k} R_{\mathfrak{p}}$ and $\operatorname{dim} R_{\mathfrak{m}} / \mathfrak{p} R_{\mathfrak{m}}=\operatorname{td}_{k} R_{\mathfrak{m}} /$ $\mathfrak{p} R_{\mathfrak{m}}$ by Corollary 4.1 .

Remark 4.2. In Theorem 4.2, let $\ell$ be a subfield of $A$ such that tr.deg $\operatorname{de}_{\ell} A$ $<\infty$. Then can we get from $\ell$ a subfield of $R$ like $k$ of Theorem 4.2? But, even if $A$ is a finite $R$-module, it seems difficult to get it. For such an example, see Example 6.2.

Let $R$ and $A$ be domains such that $R \subseteq A$. If the lying-over theorem holds between $R$ and $A$, and if the going-down theorem or the going-up theorem holds between them, then the latter part of the assumption of Theorem 4.2 is satisfied. Hence we have the following corollary.

Corollary 4.2. Let $k$ be a field, and let $R$ and $A$ be Noetherian

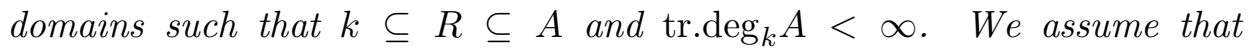
$\operatorname{dim} A_{\mathfrak{M}}=\operatorname{td}_{k} A_{\mathfrak{M}}$ for any $\mathfrak{M} \in \operatorname{Max} A$, and that $A$ is integral or faithfully flat over $R$. Then if $A$ is catenary, so is $R$, and we have $\operatorname{dim} R_{\mathfrak{m}}=\operatorname{td}_{k} R_{\mathfrak{m}}$ for any $\mathfrak{m} \in \operatorname{Max} R$. Moreover if $A$ is universally catenary, so is $R$. 
Proof. It is enough to show the latter part. Let $\underline{X}=\left\{X_{1}, \ldots, X_{n}\right\}$ be a set of variables over $A$ for any natural number $n$. Since $A[\underline{X}]$ is catenary and is integral or faithfully flat over $R[\underline{X}]$, and since $\operatorname{dim} A[\underline{X}]_{P}=\operatorname{td}_{k} A[\underline{X}]_{P}$ for any $P \in \operatorname{Max} A[\underline{X}]$ by Proposition 3.2 , we have the conclusion by the former part.

Case B The answer in Case B is negative as follows.

ExAmple 4.1. There are a Noetherian local domain $A$ and a quasilocal domain $R$ containing a field $k$ such that they satisfy the following conditions: $A$ dominates $R ; k$ is a tm-subfield of $R ; \operatorname{dim} A=\operatorname{td}_{k} A$ and $\operatorname{dim} R<\operatorname{td}_{k} R$.

Let $k$ be any field and $X, Y$ variables over $k$, and set $S=\{\alpha X \mid \alpha \in$ $k(Y)\}$. Then we have $k \subset k[S] \subset k(Y)[X]$ and $X k(Y)[X] \cap k[S]=S k[S]$. Setting $R=k[S]_{(S)}, \mathfrak{m}=(S) R, A=k(Y)[X]_{(X)}$ and $\mathfrak{n}=X A$, we have $k \subset R \subset A$ and $\mathfrak{n} \cap R=\mathfrak{m}$. Then $(R, \mathfrak{m})$ and $(A, \mathfrak{n})$ are the required domains.

Proof. It follows easily that $\operatorname{dim} A=\operatorname{td}_{k} A$ and $k$ is a tm-subfield of $R$. Now for any $\alpha \in k(Y)$, we have $(\alpha X)^{2}=X\left(\alpha^{2} X\right) \in X R$, so that $\sqrt{X R}=\mathfrak{m}$. Therefore for any $\mathfrak{p} \in \operatorname{Spec} R$ with $\mathfrak{p} \subset \mathfrak{m}$, we have $X \notin \mathfrak{p}$. Hence $\mathfrak{p} R[1 / X]$ is a proper ideal of $R[1 / X]$. For any $f \in S k[S]$ with $f \neq 0$, we write $f=\sum_{i \geq k} a_{i} X^{i}=a_{k} X^{k}\left(1+\sum_{i>k}\left(a_{i} / a_{k}\right) X^{i-k}\right)$, where each $a_{i}$ is an element of $k(Y)$ and $a_{k} \neq 0$. Since $1+\sum\left(a_{i} / a_{k}\right) X^{i-k}$ is a unit in $R$, and since $a_{k} X^{k}$ is a unit in $R[1 / X], f$ is also a unit in $R[1 / X]$, so that $\mathfrak{p}=(0)$. Therefore $\operatorname{dim} R=1<\operatorname{td}_{k} R$.

On the other hand, if $A$ is catenary and $k$ is a tm-subfield of $A$, we have an affirmative answer in Case B as follows, where $R$ need not be local.

THEOREM 4.3. Let $R$ be a domain containing a field $k$ and $(A, \mathfrak{n}) a$ Noetherian catenary local domain containing $R$, and assume that $k$ is a tm-subfield of $A$ and $\operatorname{dim} A=\operatorname{tr} \cdot \operatorname{deg}_{k} A$. Then $\mathfrak{n} \cap R \in \operatorname{Max} R$ and $\operatorname{dim} R=$ ht $\mathfrak{n} \cap R=$ tr. $\operatorname{deg}_{k} R$.

Proof. Since $A$ is catenary, we have $\operatorname{dim} A / \mathfrak{p}=\operatorname{tr} \cdot \operatorname{deg}_{k} A / \mathfrak{p}$ for any $\mathfrak{p} \in \operatorname{Spec} A$ by Theorem 3.1. Therefore we can prove this theorem in the same way as the proof of Theorem C, where Alamelu's theorem (cf. [1, Proposition 3.4]) is used. 
Remark 4.3. In Theorem 4.3, it is necessary to assume that $k$ is a tm-subfield of $A$. Concerning this, see Example 6.6.

Theorem 4.3 is a natural generalization of Theorem C. We give an example that satisfies the conditions of Theorem 4.3 as follows.

Corollary 4.3. Let $k$ be a field, $k[\underline{a}]=k\left[a_{1}, \ldots, a_{n}\right]$ a normal affine domain over $k$ and $\mathfrak{m}$ any maximal ideal of $k[\underline{a}]$, and denote the Henselization of $k[\underline{a}]_{\mathfrak{m}}$ by $A$. Then for any domain $R$ with $k \subseteq R \subseteq A$, we have $\mathfrak{m} A \cap R \in \operatorname{Max} R$ and $\operatorname{dim} R=\operatorname{tr} . \operatorname{deg}_{k} R$.

Proof. Since the completion of $A$ is equidimensional, it follows from

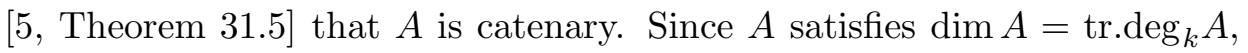
the assertion follows from Theorem 4.3.

In Theorem 4.3, we have $\operatorname{dim} R \leq \operatorname{dim} A$. But if the subfield $k$ is not a tm-subfield of $A$, it may happen that $\operatorname{dim} R>\operatorname{dim} A$ as follows.

ExAmPLE 4.2. Let $k$ be any field. For two variables $X, Y$ over $k$, $(X, Y) k[X, Y]$ and $X k[X, Y / X]$ are prime ideals of $k[X, Y]$ and $k[X, Y / X]$, respectively. Set $R=k[X, Y]_{(X, Y)}$ and $A=k[X, Y / X]_{(X)}$. From the fact that $X k[X, Y / X] \cap k[X, Y]=(X, Y) k[X, Y]$, it follows that $k \subset R \subset A$ and $A$ dominates $R$. For these local domains, we have $\operatorname{dim} A=\operatorname{td}_{k} A$ and $\operatorname{dim} R>\operatorname{dim} A$.

\section{§5. Semigroup rings over fields}

Let $k$ be any field, $X_{1}, \ldots, X_{n}$ variables over $k$, and $M$ a semigroup generated by monomials in $X_{1}, \ldots, X_{n}$. In this section, we study the semigroup ring $k[M]$ generated by $M$ over $k$. We note that the number of the monomials generating $M$ may be infinite, and that a power exponent of each variable $X_{i}$ may be negative or rational. First of all, we show that such powers are well-defined.

Let $T=\left\{X_{i, q} \mid i=1, \ldots, n, q \in \mathbf{Q}\right\}$ be a set of variables over $k, G$ the free Abelian group $\langle T\rangle$ and $H$ the subgroup $\left\langle X_{i, q}^{u} X_{i, r}^{-v} \mid u, v \in \mathbf{Z}, u q=v r\right\rangle$ of $G$. Set $I_{n}=G / H$. Then for any $w \in \mathbf{Z}$ and any $q, r \in \mathbf{Q}$ we have $X_{i, q}^{w}=X_{i, q w}$ and $X_{i, q} X_{i, r}=X_{i, q+r}$ in $I_{n}$. Hence for an element $Y \in I_{n}$, $Y^{w}=X_{i, 1}$ for some $i$ and some $w \in \mathbf{Q}-\{0\}$ if and only if $Y=X_{i, 1 / w}$. Therefore in $I_{n}$ we denote $X_{i, 1}$ by $X_{i}$, and $X_{i, q}$ by $X_{i}^{q}$ for any $q \in \mathbf{Q}$. Then $I_{n}=\left\langle X_{i}^{q} \mid q \in \mathbf{Q}, i=1, \ldots, n\right\rangle$, so that the power exponents of variables may be rational numbers. Then we have the following theorem. 
THEOREM 5.1. Let $k$ be any field and $M$ a subsemigroup of $I_{n}$. Then we have $\operatorname{dim} k[M]=\operatorname{tr} \cdot \operatorname{deg}_{k} k[M]$, and we can construct a chain of prime ideals of length $\operatorname{dim} k[M]$. Moreover if $M \subseteq\left\langle X_{i}^{q}\right| q \in \mathbf{Q}, q>0, i=$ $1, \ldots, n\rangle$, we can construct such a chain descending from $M k[M]$.

Proof. Set $t=\operatorname{tr} \cdot \operatorname{deg}_{k} k[M]$. Then we show that $\operatorname{dim} k[M]=t$ by induction on $t$. The proof shows us how to construct a chain of prime ideals of length $t$. First of all, according to [4], we define a map $\log _{X}$ : $M \longrightarrow \mathbf{Q}^{n}$ by $\log _{X} X_{1}^{a_{1}} \cdots X_{n}^{a_{n}}=\left(a_{1}, \ldots, a_{n}\right)$. Since $t=\operatorname{dim}_{\mathbf{Q}} \mathbf{Q}\left(\log _{X} M\right)$, there exist $m_{1}, \ldots, m_{t-1} \in M$ such that $\log _{X} m_{1}, \ldots, \log _{X} m_{t-1}$ are linearly independent over $\mathbf{Q}$. Set $\left(a_{i 1}, \ldots, a_{i n}\right)=\log _{X} m_{i}$ for each $i$. Then we may assume that

$$
D=\left|\begin{array}{ccc}
a_{11} & \cdots & a_{1 t-1} \\
\cdots & \cdots & \cdots \\
a_{t-11} & \cdots & a_{t-1 t-1}
\end{array}\right| \neq 0
$$

For any natural numbers $s_{t}, \ldots, s_{n}$, let us consider the following determinant:

$$
D_{s}=\left|\begin{array}{ccc}
a_{11} & \cdots & a_{1 t-1}+\sum s_{j} a_{1 j} \\
\cdots & \cdots & \cdots \\
a_{t-11} & \cdots & a_{t-1 t-1}+\sum s_{j} a_{t-1 j}
\end{array}\right|=D+\sum s_{j} D_{j}
$$

where each $D_{j}$ is a rational number. Since $D \neq 0$, there exist natural numbers $s_{t}, \ldots, s_{n}$ such that $D_{s} \neq 0$. Set $U=\mathbf{Q}$ in the former case of the theorem or $U=\{q \in \mathbf{Q} \mid q>0\}$ in the latter case. Then setting $S_{v}=$ $\left\{X_{i}^{q} \mid q \in U, i=1, \ldots, v\right\}$ for $1 \leq v \leq n$, we get the $k$-homomorphism $f: k\left[S_{n}\right] \longrightarrow k\left[S_{t-1}\right]$ such that $f\left(X_{i}^{q}\right)=X_{i}^{q}, f\left(X_{j}^{q}\right)=X_{t-1}^{q s_{j}}$ for any $q \in U$ and any $i, j$ with $i<t \leq j$, where $X_{0}=1$ in the former case of the theorem and $X_{0}=0$ in the latter case. Set $g=\left.f\right|_{k[M]}$ and $M^{\prime}=g(M)$. Then the $k$ homomorphism $g: k[M] \longrightarrow k\left[M^{\prime}\right]$ is surjective, and Ker $g=\left(\left\{X_{j}^{q}-X_{t-1}^{q s_{j}} \mid\right.\right.$ $q \in U, t \leq j \leq n\}) k\left[S_{n}\right] \cap k[M]$. In the latter case we have Ker $g \subseteq M k[M]$. Since tr. $\operatorname{deg}_{k} k\left[M^{\prime}\right]=\operatorname{dim}_{\mathbf{Q}} \mathbf{Q}\left(\log _{X} M^{\prime}\right)=t-1$, it holds that Ker $g \supset(0)$. Since $\operatorname{dim} k[M] \leq t$, and since $\operatorname{dim} k\left[M^{\prime}\right]=t-1$ by induction, we have ht $\operatorname{Ker} g=1$, so that $\operatorname{dim} k[M]=t$.

ExAmPLE 5.1. Let $k$ be any field and $M$ the semigroup generated by $\left\{X Y^{q} \mid q \in \mathbf{Q}, q \geq 0\right\}$, where $X, Y$ are variables over $k$. Then $\operatorname{dim} k[M]=$ tr. $\operatorname{deg}_{k} k[M]=2$.

We construct a chain of prime ideals descending from $(M)$ such that the length is $\operatorname{dim} k[M]$. Since $\operatorname{dim}_{\mathbf{Q}} \mathbf{Q}\left(\log _{X} M\right)=2$, we have $t=2$ in the 
proof of Theorem 5.1 , so that we can substitute $m_{1}$ and $s_{2}$ by $(1,0)$ and 1 , respectively. Therefore

$$
\begin{aligned}
\operatorname{Ker} g & =\left(\left\{Y^{q}-X^{q} \mid q \in U\right\}\right) k\left[S_{2}\right] \cap k[M] \\
& =\left(\left\{X Y^{j+1}-X^{2} Y^{j} \mid j \in \mathbf{Q}, j \geq 0\right\}\right) k[M] .
\end{aligned}
$$

Hence $(0) \subset \operatorname{Ker} g \subset(M)$ is a chain of prime ideals of length 2 .

\section{§6. Examples}

ExAmPLE 6.1. There is a Noetherian local domain $A$ such that for any natural number $u$, there is a maximal subfield $L$ of $A$ with $\operatorname{tr} . \operatorname{deg}_{L} A=u$.

Let $k$ be a field, $X_{1}, \ldots, X_{m}, \ldots, Z$ variables over $k$ and $v$ any natural number. Set $K=k\left(X_{1}, \ldots, X_{m}, \ldots\right), A=K[Z]_{(Z)}, \xi_{i}=X_{i}+X_{i+v} Z(i=$ $1,2, \ldots)$ and $L=k\left(\xi_{1}, \ldots, \xi_{m}, \ldots\right)$. Then $K$ and $L$ are maximal subfields of $A$, and tr. $\operatorname{deg}_{K} A=1$ and $\operatorname{tr} \cdot \operatorname{deg}_{L} A=v+1$. Hence $A, K$ and $L$ are the required set of domain and subfields.

Proof. We have only to consider the field $L$. For each $i$, we have $\xi_{i} \equiv X_{i} \bmod Z A$, so that $\xi_{1} \bmod Z A, \ldots, \xi_{m} \bmod Z A, \ldots$ are algebraically independent over $k$. Hence $k\left[\xi_{1}, \ldots, \xi_{m}, \ldots\right] \cap Z A=(0)$, so that $L \subset A$. Since $L+Z A / Z A \cong K, L$ is a maximal subfield of $A$.

Now since $\xi_{1}, \ldots, \xi_{v} \in L\left(X_{1}, \ldots, X_{v}, Z\right)$, we have $X_{1+v}, \ldots, X_{2 v} \in$ $L\left(X_{1}, \ldots, X_{v}, Z\right)$. In the same way, we have $X_{1+2 v}, \ldots \in L\left(X_{1}, \ldots, X_{v}, Z\right)$, so that $L\left(X_{1}, \ldots, X_{v}, Z\right)=Q(A)$. Hence it is enough to show that $X_{1}, \ldots$, $X_{v}, Z$ are algebraically independent over $L$. For the purpose, putting $L_{t}=k\left(\xi_{1}, \ldots, \xi_{t}\right)$ for each natural number $t$, we have only to prove that tr. $\operatorname{deg}_{L_{t}} L_{t}\left(X_{1}, \ldots, X_{v}, Z\right)=v+1$. Since $L_{t}\left(X_{1}, \ldots, X_{v}, Z\right)=k\left(X_{1}, \ldots\right.$, $\left.X_{t+v}, Z\right)$, we have tr. $\operatorname{deg}_{k} L_{t}\left(X_{1}, \ldots, X_{v}, Z\right)=t+v+1$, so that we come to the conclusion.

Using Example 6.1, we can get the example stated in Remark 4.2 as follows.

Example 6.2. There are Noetherian local domains $A$ and $R$ satisfying the following conditions: $A$ is a finite $R$-module; $\operatorname{tr} \cdot \operatorname{deg}_{L} A<\infty$ and tr. $\operatorname{deg}_{L \cap R} R=\infty$ for a maximal subfield $L$ of $A$.

Under the notation of Example 6.1, we assume further that the characteristic of $k$ is 0 . For any natural number $n$ with $n \geq 2$, set $R=K\left[Z^{n}\right]_{\left(Z^{n}\right)}$. Then $A, L$ in Example 6.1 and $R$ are the required set of domains and field. 
Proof. It is enough to show that $L \cap K\left(Z^{n}\right)=k$. Let $\alpha$ be any element of $L \cap K\left(Z^{n}\right)$ with $\alpha \neq 0$. Then there exists a natural number $t$ such that $L_{t} \cap K\left(Z^{n}\right) \ni \alpha$, where $L_{t}$ is the field defined in the proof of Example 6.1. Set $\alpha=f / g=u / v$, where $f=f\left(\xi_{1}, \ldots, \xi_{t}\right), g=g\left(\xi_{1}, \ldots, \xi_{t}\right) \in k\left[\xi_{1}, \ldots, \xi_{t}\right]$ and $u=u\left(Z^{n}\right), v=v\left(Z^{n}\right) \in K\left[Z^{n}\right]$. We may assume that $f, g$ and $u, v$ are relatively prime in $k\left[\xi_{1}, \ldots, \xi_{t}\right]$ and in $K\left[Z^{n}\right]$, respectively. Then $v f=$ $u g$. (*) We consider $f, g, u$ and $v$ as elements of $K[Z]$, and compare the terms of degree 0 and 1 in $Z$ on both sides of $(*)$. Then we have the following equations:

$$
\begin{aligned}
& v(0) f\left(X_{1}, \ldots, X_{t}\right)=u(0) g\left(X_{1}, \ldots, X_{t}\right), \\
& v(0)\left(X_{1+v} f_{1}+\cdots+X_{t+v} f_{t}\right)=u(0)\left(X_{1+v} g_{1}+\cdots+X_{t+v} g_{t}\right),
\end{aligned}
$$

where $f_{i}=\frac{\partial f}{\partial \xi_{i}}\left(X_{1}, \ldots, X_{t}\right), g_{i}=\frac{\partial g}{\partial \xi_{i}}\left(X_{1}, \ldots, X_{t}\right) \quad(i=1, \ldots, t)$. Since $u$ and $v$ are relatively prime in $K\left[Z^{n}\right]$, it follows that $u(0) \neq 0$ or $v(0) \neq 0$, so that by (1) and (2) we have

$$
\begin{aligned}
& g\left(X_{1}, \ldots, X_{t}\right)\left(X_{1+v} f_{1}+\cdots+X_{t+v} f_{t}\right) \\
& \quad=f\left(X_{1}, \ldots, X_{t}\right)\left(X_{1+v} g_{1}+\cdots+X_{t+v} g_{t}\right) .
\end{aligned}
$$

Comparing the coefficients of $X_{t+v}$ on both sides of (3), we have $g\left(X_{1}, \ldots\right.$, $\left.X_{t}\right) f_{t}=f\left(X_{1}, \ldots, X_{t}\right) g_{t}$. Since $f$ and $g$ are relatively prime in $k\left[\xi_{1}, \ldots, \xi_{t}\right]$, we have $f\left(X_{1}, \ldots, X_{t}\right)\left|f_{t}, g\left(X_{1}, \ldots, X_{t}\right)\right| g_{t}$. Therefore $f_{t}=g_{t}=0$, so that $f, g \in k\left[\xi_{1}, \ldots, \xi_{t-1}\right]$, that is, $\alpha \in L_{t-1} \cap K\left(Z^{n}\right)$. Hence by induction on $t$, we have $\alpha \in k$.

Example 6.3. There is a Noetherian domain $A$ containing a field $k$ such that $\operatorname{td}_{k} A>\max \left\{\operatorname{td}_{k} A_{\mathfrak{m}} \mid \mathfrak{m} \in \operatorname{Max} A\right\}$.

Let $k$ be any algebraically closed field, and for each $a \in k$ set $f_{a}(X, Y)=$ $X+a Y+a^{2}$, where $X$ and $Y$ are variables over $k$. Let $S$ be the multiplicatively closed subset generated by $\left\{f_{a}(X, Y) \mid a \in k\right\}$, and set $A=$ $S^{-1} k[X, Y]$. Then $A$ and $k$ are the required pair of domain and field.

Proof. First, we note that $f_{a}(X, Y)$ is irreducible over $k$ for any $a \in k$. Moreover we find that for any $\mathfrak{m} \in \operatorname{Max} k[X, Y]$ there exists $a \in k$ such that $f_{a}(X, Y) \in \mathfrak{m}$. In fact, since $k$ is algebraically closed, there exist $u, v \in k$ such that $\mathfrak{m}=(X-u, Y-v)$, and for the elements $u$ and $v$ there exists $a \in k$ such that $f_{a}(u, v)=0$. Hence $f_{a}(X, Y) \in \mathfrak{m}$. From this it follows that $\operatorname{dim} A \leq 1$. 
It is enough to show that $k$ is a maximal subfield of $A$. We assume the contrary. Then there exists an element $T \in A-k$ such that $k(T) \subseteq A$. We can write $T=a Q / P$, where $a \in k, P, Q \in S$ and $P, Q$ are relatively prime. Let $n$ and $m$ be the numbers of the prime factors of $P$ and $Q$, respectively. Replacing $a^{-1} T$ by $T$, we may assume that $a=1$. Moreover replacing $T^{-1}$ by $T$ if necessary, we may assume that $m \leq n$. Now we have $T-1=b Q^{\prime} / P^{\prime}$, where $b \in k, P^{\prime}, Q^{\prime} \in S$ and $P^{\prime}, Q^{\prime}$ are relatively prime. Since $T-1=(Q-P) / P$, we have

$$
Q-P=c Q^{\prime} \text { for some } c \in k \text {. }
$$

Let $\ell$ be the number of the prime factors of $Q^{\prime}$. If $m=n$, we have $\ell<n$, so that replacing $T-1$ by $T$, we may assume that $m<n$. Then by (4), we have $\ell=n, c=-1$. Put

$$
P=f_{a_{1}} \cdots f_{a_{n}}, Q^{\prime}=f_{b_{1}} \cdots f_{b_{n}},
$$

and for any $t \in k$ substitute $X$ by $t Y$ on the both sides of (4). Then comparing the terms of degree $n$ in $Y$, we have

$$
\left(t+a_{1}\right) \cdots\left(t+a_{n}\right)=\left(t+b_{1}\right) \cdots\left(t+b_{n}\right) .
$$

Since this holds for any $t \in k$, there exists a permutation $\sigma$ of $1, \ldots, n$ such that $a_{i}=b_{\sigma(i)}$ for all $i$, so that $P=Q^{\prime}$. This is a contradiction.

Remark 6.1. From the example above, we find that there exists a Noetherian domain $A$ containing a field $k$ such that $\operatorname{dim} A_{\mathfrak{m}}=\operatorname{td}_{k} A_{\mathfrak{m}}$ for any $\mathfrak{m} \in \operatorname{Max} A$, but $\operatorname{dim} A<\operatorname{td}_{k} A$.

EXAMPLE 6.4. For the conditions (a) and (b) of Theorem 3.1, there is a Noetherian local domain which satisfies (a) but does not satisfy (b).

Fortunately, using [6, p.203, Example 2], we can construct such an example. First of all, we state Nagata's construction. For this example we have only to use the case $m=0$ of Nagata's construction. But in the following examples, we need the case $m \geq 1$. Hence we state the construction in all the cases.

Let $k$ be a field, $X$ a variable over $k$ and $z_{1}, \ldots, z_{s}$ elements of $k[[X]]$ such that $z_{1}, \ldots, z_{s}$ are algebraically independent over $k(X)$. For each $z_{i}=$ $\sum a_{i j} X^{j} \quad\left(a_{i j} \in k\right)$, put $z_{i j}=\left(z_{i}-\sum_{h<j} a_{i h} X^{h}\right) / X^{j-1} \quad(j=1,2, \cdots)$. Let $A$ be the ring generated over $k$ by $X$ and by all the $z_{i j}$ 's, and put 
$B=A\left[Y_{1}, \ldots, Y_{m}\right]$, where $Y_{1}, \ldots, Y_{m}$ are variables over $A$. Moreover let $\mathfrak{m}$ and $\mathfrak{n}$ be the maximal ideals of $B$ generated by $X, Y_{1}, \ldots, Y_{m}$ and by $X-1, z_{1}, \ldots, z_{s}, Y_{1}, \ldots, Y_{m}$, respectively. Put $C=S^{-1} B$, where $S=B-$ $\mathfrak{m} \cup \mathfrak{n}$, and put $I=\mathfrak{m} C \cap \mathfrak{n} C$ and $R=k+I$. Then $R$ is a Noetherian local domain with $\operatorname{dim} R=s+m+1$, and $C$ is a finite $R$-module. Since $R / I \cong k, k$ is a maximal subfield of $R$. Moreover since $Q(R)=Q(B)=$ $k\left(X, z_{1}, \ldots, z_{s}, Y_{1}, \ldots, Y_{m}\right)$, we have $\operatorname{dim} R=\operatorname{tr} \cdot \operatorname{deg}_{k} R$. It is known that if $m=0, R$ is catenary but is not universally catenary, and if $m>0, R$ is not catenary.

Now set $m=0$. Then, for a variable $U$ over $R$ and for some $\mathfrak{M} \in$ $\operatorname{Max} R[U], R[U]_{\mathfrak{M}}$ is the required local domain.

Proof. Since $R$ is a catenary local domain with $\operatorname{dim} R=\operatorname{td}_{k} R$, we have $\operatorname{dim} R_{\mathfrak{p}}=\operatorname{td}_{k} R_{\mathfrak{p}}$ for any $\mathfrak{p} \in \operatorname{Spec} R$ by Theorem 3.1. Hence it follows from Proposition 3.2 that $\operatorname{dim} R[U]_{\mathfrak{P}}=\operatorname{td}_{k} R[U]_{\mathfrak{P}}$ for any $\mathfrak{P} \in \operatorname{Spec} R[U]$. On the other hand, since $R$ is not universally catenary, $R[U]$ is not catenary by [5, Theorem 31.7]. Hence by Theorem 3.1, there exist $\mathfrak{M} \in \operatorname{Max} R[U]$ and $\mathfrak{P} \in \operatorname{Spec} R[U]$ with $\mathfrak{P} \subseteq \mathfrak{M}$ such that $\operatorname{dim} R[U]_{\mathfrak{M}} / \mathfrak{P} R[U]_{\mathfrak{M}}<\operatorname{td}_{k} R[U]_{\mathfrak{M}} /$ $\mathfrak{P} R[U]_{\mathfrak{M}}$. Therefore $R[U]_{\mathfrak{M}}$ is the required example.

Remark 6.2. In Nagata's construction above, we have $\operatorname{dim} B_{\mathfrak{m}}=m+1$, and since $k$ is a maximal subfield of $B_{\mathfrak{m}}$, we have $\operatorname{td}_{k} B_{\mathfrak{m}}=s+m+1$. Hence we find that for any natural number $s$ there exists a Noetherian local domain $R$ containing a field $k$ such that $\operatorname{td}_{k} R-\operatorname{dim} R=s$.

EXAMPLE 6.5. There exist a Noetherian local domain $(R, \mathfrak{m})$ and a


$\operatorname{dim} R=\operatorname{td}_{k} R$; for some $\mathfrak{p} \in \operatorname{Spec} R, \operatorname{dim} R_{\mathfrak{p}}<\operatorname{td}_{k} R_{\mathfrak{p}}$ and $\operatorname{dim} R / \mathfrak{p}=$ $\operatorname{td}_{k} R / \mathfrak{p}$.

In Nagata's construction above, let us consider the case $m \geq 1$. Then for any $\mathfrak{p} \in \operatorname{Spec} R$ it holds that

(1) if some $\mathfrak{P} \in \operatorname{Spec} C$ with $\mathfrak{P} \not \supset X$ lies over $\mathfrak{p}$, we have $\operatorname{dim} R_{\mathfrak{p}}=\operatorname{td}_{k} R_{\mathfrak{p}}$, and in the other case we have $\operatorname{dim} R_{\mathfrak{p}}+s=\operatorname{td}_{k} R_{\mathfrak{p}}$;

(2) if some $\mathfrak{P} \in \operatorname{Spec} C$ with $\mathfrak{P} \ni X$ or $\mathfrak{P} \subseteq \mathfrak{n} C$ lies over $\mathfrak{p}$, we have $\operatorname{dim} R / \mathfrak{p}=\operatorname{td}_{k} R / \mathfrak{p}$, and in the other case we have $\operatorname{dim} R / \mathfrak{p}+s=$ $\operatorname{td}_{k} R / \mathfrak{p}$. 
In particular, we find that $R$ is the required local domain.

Proof. (1) If some $\mathfrak{P} \in \operatorname{Spec} C$ with $\mathfrak{P} \not \supset X$ lies over $\mathfrak{p}, C_{\mathfrak{P}}$ is a localization of $C\left[X^{-1}\right] \cong S^{-1} k\left[X, X^{-1}, z_{1}, \ldots, z_{s}, Y_{1}, \ldots, Y_{m}\right]$ and $R_{\mathfrak{p}}$ is dominated by $C_{\mathfrak{P}}$. Therefore it follows from Corollary 3.2 and 4.1 that $\operatorname{dim} R_{\mathfrak{p}}=\operatorname{td}_{k} R_{\mathfrak{p}}$. If $\mathfrak{P} \ni X$ for every $\mathfrak{P} \in \operatorname{Spec} C$ lying over $\mathfrak{p}$, we have $\operatorname{dim} R / \mathfrak{p}=m+1-$ ht $\mathfrak{p}$ by the proof of $\left[6\right.$, p.203, Example 2]. Since $\operatorname{dim} R / \mathfrak{p}=\operatorname{td}_{k} R / \mathfrak{p}$ by (2) below, it follows from Lemma 3.1 that

$$
\operatorname{td}_{k} R_{\mathfrak{p}}=\operatorname{td}_{k} R-\operatorname{td}_{k} R / \mathfrak{p}=\operatorname{dim} R-\operatorname{dim} R / \mathfrak{p}=s+\operatorname{dim} R_{\mathfrak{p}}
$$

(2) If some $\mathfrak{P} \in \operatorname{Spec} C$ with $\mathfrak{P} \ni X$ lies over $\mathfrak{p}, C / \mathfrak{P}$ is a homomorphic image of $C / X C \cong B_{\mathfrak{m}} / X B_{\mathfrak{m}} \cong k\left[Y_{1}, \ldots, Y_{m}\right]_{\left(Y_{1}, \ldots, Y_{m}\right)}$. Since $C / \mathfrak{P}$ dominates $R / \mathfrak{p}$, we have $\operatorname{dim} R / \mathfrak{p}=\operatorname{td}_{k} R / \mathfrak{p}$ by Corollary 4.1. If some $\mathfrak{P} \in \operatorname{Spec} C$ with $\mathfrak{P} \subseteq \mathfrak{n} C$ lies over $\mathfrak{p}, C_{\mathfrak{n} C} / \mathfrak{P} C_{\mathfrak{n} C}$ is a homomorphic image of $C_{\mathfrak{n} C} \cong B_{\mathfrak{n}} \cong k\left[X, z_{1}, \ldots, z_{s}, Y_{1}, \ldots, Y_{m}\right]_{\left(X-1, z_{1}, \ldots, z_{s}, Y_{1}, \ldots, Y_{m}\right)}$. Since $C_{\mathfrak{n} C} / \mathfrak{P} C_{\mathfrak{n} C}$ dominates $R / \mathfrak{p}$, we have $\operatorname{dim} R / \mathfrak{p}=\operatorname{td}_{k} R / \mathfrak{p}$ by Corollary 4.1.

Let us assume that $\mathfrak{P} \not \supset X$ and $\mathfrak{P} \nsubseteq \mathfrak{n} C$ for any $\mathfrak{P} \in \operatorname{Spec} C$ lying over $\mathfrak{p}$. Then we have $\operatorname{dim} R / \mathfrak{p}=m+1-$ ht $\mathfrak{p}$ by the proof of $[6, p .203$, Example 2]. Since $\mathfrak{P} \not \supset X$, it follows from Lemma 3.1 and (1) above that

$$
\operatorname{td}_{k} R / \mathfrak{p}=\operatorname{td}_{k} R-\operatorname{td}_{k} R_{\mathfrak{p}}=\operatorname{dim} R-\operatorname{dim} R_{\mathfrak{p}}=s+\operatorname{dim} R / \mathfrak{p} .
$$

Remark 6.3. (1) In Example 6.5, there exists a prime ideal $\mathfrak{p}$ of $R$ such that $\operatorname{dim} R / \mathfrak{p}+s=\operatorname{td}_{k} R / \mathfrak{p}$. Hence the example above is not one which satisfies the condition (b) of Theorem 3.1 but does not satisfy the condition (a).

(2) In Example 6.5, for any natural number $m$ with $m \geq 2$ there is a maximal saturated chain of prime ideals $\mathfrak{p}_{0} \subset \mathfrak{p}_{1} \subset \cdots \subset \mathfrak{p}_{m+1}$ in $R$ such that for an integer $p$ with $1 \leq p<m$ we have $\delta\left(\mathfrak{p}_{i}\right)=0, \varepsilon\left(\mathfrak{p}_{i}\right)=s$ for any $i$ with $1 \leq i \leq p$, and $\delta\left(\mathfrak{p}_{j}\right)=s, \varepsilon\left(\mathfrak{p}_{j}\right)=0$ for any $j$ with $p<j \leq m$, where $\delta(\mathfrak{p})$ and $\varepsilon(\mathfrak{p})$ are the symbols used in Corollary 3.1.

ExAmple 6.6. There are a Noetherian semi-local domain $R$, a tmsubfield $k$ of $R$ and a Noetherian catenary local domain $(A, \mathfrak{n})$ containing $R$ such that $\operatorname{dim} A=\operatorname{td}_{k} A, \mathfrak{n} \cap R \in \operatorname{Max} R$ but $\operatorname{dim} R<\operatorname{td}_{k} R$.

We also use Nagata's example cited in Example 6.4. We assume that $m \geq 1$. For a prime ideal $\mathfrak{p}=\left(X-1, z_{1}, \ldots, z_{s}\right)$ of $B$ set $D=T^{-1} B$, where $T=B-\mathfrak{m} \cup \mathfrak{p}$. Then we have $k \subset D \subset B_{\mathfrak{p}}$, and these are the required set of domains and field. 
Proof. Since $B_{\mathfrak{p}}$ is a localization of $k\left[X, X^{-1}, z_{1}, \ldots, z_{s}, Y_{1}, \ldots, Y_{m}\right]$, we have $\operatorname{dim} B_{\mathfrak{p}}=\operatorname{td}_{k} B_{\mathfrak{p}}=s+1$. On the other hand, since $k$ is a maximal subfield of $B_{\mathfrak{m}}, k$ is also one of $D$, so that we have $\operatorname{td}_{k} D=s+m+1$. Since $\operatorname{dim} B_{\mathfrak{m}}=m+1$, we have $\operatorname{dim} D=\max \{s, m\}+1<\operatorname{td}_{k} D$.

\section{REFERENCES}

[1] S. Alamelu, Subrings of affine rings, J. Indian Math. Soc., 42 (1978), 203-214.

[2] W. Bruns and J. Herzog, Cohen-Macaulay rings, Cambridge University Press, Cambridge, 1993.

[3] R. Gilmer, B. Nashier and W. Nichols, The prime spectra of subalgebras of affine algebras and their localizations, J. Pure Appl. Algebra, 57 (1989), 47-65.

[4] M. Hochster, Rings of invariants of tori, Cohen-Macaulay rings generated by monomials, and polytopes, Ann. of Math., 96 (1972), 318-337.

[5] H. Matsumura, Commutative Ring Theory, Cambridge University Press, Cambridge, 1986.

[6] M. Nagata, Local Rings, Interscience, New York, 1962.

[7] N. Onoda and K. Yoshida, On noetherian subrings of an affine domain, Hiroshima Math. J., 12 (1982), 377-384.

Department of Mathematics

Faculty of Education and Culture

Miyazaki University

Miyazaki 889-2192, Japan

e03103u@cc.miyazaki-u.ac.jp 